\title{
Improving the transport of urgent specimens to an off-site laboratory using a novel sticker-tracker
}

\author{
Afsoon Sepahzad, Florence Ejiofor, Susan Giles, Robert Klaber
}

\begin{abstract}
Obtaining results for urgent microbiology specimens in an efficient manner is imperative to ensure that patients receive appropriate antibiotic therapy. A previous audit carried out in the Paediatric department of a central teaching hospital and a number of clinical incidents, highlighted a delay in transport of specimens (exceeding eight hours) and 'missing' specimens. This results in empirical antibiotic treatment of infection, with delay in confirming microbiology result and unnecessary, distressing repeat investigation. As an initial step we sought staff opinion to further explore the problem. A sticker was designed for the microbiology specimen bag to assign accountability, track each step in the transport process and to raise awareness of the problem. The sticker required the member of staff responsible at each stage of the process to time, date and sign it, to allow tracking of potential delays. The new sticker tracking system was promoted and launched in the Paediatric department. Initial challenges included lack of awareness of the protocol and lack of pods for sending urgent specimens. The team met regularly and completed stickers were analysed weekly to identify on-going issues and to devise solutions. Consequently total transport time was reduced to an average of 69 minutes by September (within $4 \mathrm{hr}$ target). Our intervention improved the efficiency and reliability of urgent specimen transport. This is likely to result in safer antibiotic use and avoid the need for repeat investigation. The system is now also used in the Neonatal department and has lead to the development of a new 'Central Specimens Reception'.
\end{abstract}

\section{Problem}

Obtaining microbiology specimens from infants and children can be a challenging task. Not only is it distressing for the patient and parents, but the process involves a lot of time, and preparation, from the clinical team. In young infants, sedation is sometimes used to perform lumbar puncture in difficult cases. A delay in receiving microbiology results can impact clinical management, leading to empirical antibiotic treatment of infection and unnecessary, distressing repeat investigation. All microbiology specimens are sent to an off-site laboratory for analysis. With multiple steps in the transport process involving clinicians, porters, and laboratory staff, the pathway is complex with multiple potential causes for the delay in obtaining results. All stakeholders involved were keen to investigate and improve this vital service.

\section{Background}

The project was carried out in the Paediatric Department at a Central London teaching hospital. The Paediatric Department comprises four children's wards with over forty beds, the Paediatric Intensive Care Unit and the Paediatric Accident and Emergency. A departmental audit highlighted a delay in this transport process with some samples taking in excess of eight hours to reach the laboratory and others reported as 'missing'. The audit did not highlight where delays were taking place or possible contributing factors.

The protocol for sending microbiology specimens detailed the steps involved in sending specimens both in and out-of-hours with emphasis on the use of the Pneumatic Tube System (PTS) for sending specimen in-hours. With limited knowledge of the protocol among staff in the department, and other limitations which were later realised, transport of urgent specimens was not as efficient as it should be. The issue had also been highlighted through clinical incident reporting. Although an important problem, with possible implications to patient safety, there was limited information on where delays were occurring and areas in which improvements could be made. Consequently there had been little in the way of progress to improve the service.

\section{Baseline Measurement}

Details of total transit time obtained from a previous departmental audit, opinion gauged from staff on the ward and clinical incident forms were the main drivers for this project. A sticker was designed to affix to microbiology specimen bags. This served several purposes: To assign accountability to those involved, to track each step in the transport process and to raise awareness. The multipurpose sticker allowed us to assess the scale of the problem and provided an objective way of monitoring improvement. By asking staff to sign, time and date at each step we were able to record the exact time taken at each stage in order observe potential areas which could be causing delay in transport. Our baseline measurement was obtained one month after introducing the sticker tracker which revealed an average transport time of 160 minutes.

See supplementary file: Figure 1- The Sticker Tracker.doc

\section{Design}

It was imperative that our intervention allowed us to identify exactly 
where delays in the transport process were occurring so that these could be used as a platform to explore potential contributing factors. The Quality Improvement Project team comprised of a Consultant Paediatrician, the Quality and Safety Manager of Pathology Business Services, the Head of the Paediatric Risk and Audit Team, and a Foundation Year 1 Doctor. Together we considered various methods of tracking the transport process considering financial limitations and sustainability, finally deciding on the use of a sticker tracking system.

Using the protocol for sending urgent microbiology specimens a sticker was designed which required the member of staff responsible at each stage of the process to time, date and sign, at each stage of the process (Figure 1). On reviewing the design we further agreed we would number the stickers making note of which number stickers were allocated to the various clinical areas, enabling us to also monitor use of the stickers in the different Paediatric departments. The production of the stickers was funded by the off-site Microbiology Laboratory . In the initial planning stages we decided that the stickers would be employed for a six month period allowing us enough time to assess the problem, make necessary changes and monitor any improvement to the service, after which time the sticker would no longer need to be in place.

We anticipated that asking staff to complete more paperwork would be a challenge and that, in the context of a busy working environment, staff may forget to use the stickers so we ensured that we dedicated time to appropriately promote the use of the stickers using emails, induction meetings, posters and meeting staff in person. Following the launch, all completed stickers were analysed weekly using an Excel spreadsheet.

\section{Strategy}

In the initial phase of auditing stickers and liaising with staff, key problem areas were highlighted. These included lack of knowledge of the protocol for sending urgent specimens, lack of trust in the Pneumatic Tube System (PTS) which should be used for sending specimens in-hours, insufficient pods to send samples via the PTS and poor completion of stickers. These led to a delay in samples reaching the chemical pathology lab. Porters were called to deliver specimens via foot which took longer and was an inappropriate use of the portering service, taking porters away from other duties in the hospital.

We subsequently improved the design of the protocol, so that is was more user-friendly, and were displayed in appropriate ward locations.

We provided education of the protocol at a local level to nurses and doctors. An audit was conducted on the pneumatic tube system, which tracked transit time of pods from the ward, to the Chemical Pathology Lab, with a view to increasing staff trust the pod system. Proposals were made for obtaining funding for the provision of more pods which has been granted. The stickers were further promoted at new doctors' induction, meetings with the portering department and laboratory staff, to encourage correct completion of the sticker.

In the second phase we observed more promising results with an improved rate of sticker completion and an improved specimen transport time ranging from 50 minutes to 4.5 hours. The reporting of three incidents via Datix (online incident reporting software) caused us to re-evaluate and make further changes. The three incidents were investigated at senior departmental level using rootcause analysis. Following this further strategies were implemented which included pathology staff from the laboratory taking on the additional responsibility of chasing samples which have not reached their laboratory within 2 hours of receiving a call from the ward stating that a sample has been sent, the use of one portering system to be used in and out-of-hours, a 'Pod Awareness Campaign' reviving the use of the Pneumatic Tube System, and most significantly the development of a new Central Specimen Reception. Previously samples were sent to different locations depending on whether they were sent in-hours or out-of-hours. The centralisation of a specimen collection point which would be managed by laboratory staff will hopefully prevent samples not reaching the correct location and enable more efficient transfer to the off-site laboratory.

\section{Post-Measurement}

Measurement of improvement was through continual audit of completed stickers on a weekly basis. Between March and September we collected a total of 51 stickers, 41 of which were completed with enough information to include in the analysis. From March 2012 to September 2012 we saw a dramatic reduction in total transit time from an average of 160 minutes in March 2012 to an average of 69 minutes by September (Figure 2). This demonstrated objective improvement in transport of urgent specimens with the faster identification of causative organisms leading to more appropriate use of antibiotic therapy and prevention of the need for repeat investigation. There has also been a reduction in the total number of Datix incidents reported regarding delayed results or missing samples. Clinical staff have also reported greater reliance in the service, with better understanding of the protocol. The sticker tracker has been employed by the Neonatal Department and other adult medical departments have expressed desire to use the sticker. Our intervention successfully identified problem areas allowing implementation of changes. We hope that the intervention has resulted in a change in culture in those using the service.

See supplementary file: Figure 2- Average Specimen Transit Time.ppt

\section{Lessons and Limitations}

Despite attempts at promoting the new tracking system, we found that initial use of the stickers were limited. This demonstrated the importance of thorough planning and promotion before the introduction of any intervention and the need to employ various methods of communication to do this. We found meeting with groups of professionals in person was most helpful in explaining the importance of involvement in the project. We also recognised the importance of involving multiple stakeholders in any quality improvement project in order to gain a better perspective of the 
problem. Another limitation was funding which affected our ability to provide more pods for use in the Pneumatic Tube System at an early stage.

The project highlighted the complexity of one single service in the large hospital setting and how one service and its failure can impact on patient care. It is to engage multiple stakeholders, and a large team, to achieve a successful outcome.

Staff need to be informed of protocols in their local department, whether they are clinical, procedural or for the use of a service as ultimately better knowledge of service leads to better use of it. Most importantly we were reminded of the responsibility we all have in maintaining patient safety and the importance of regular involvement in quality improvement at any level in training.

\section{Conclusion}

The transport of urgent specimens to an off-site laboratory is a complex service involving multiple members of staff and a number of steps. Multiple factors were the cause of the delay in the transport of urgent specimen, compromising patient safety. A simple sticker tracker was employed highlighting specific problems which could be addressed. The measures implemented dramatically reduced specimen transport time and have led to positive changes in the service leading to improved patient care. The intervention acted as a reminder of the protocol and raised awareness of an important issue in the department.

The methodology employed here can be applied to other situations where the cause of the problem is not immediately identifiable. Involving multiple stakeholders will provide greater perspective of any problem and opitimise the chances of a successful outcome. The development of a new central specimen reception will hopefully contribute in improving the problem throughout the hospital. For the outcome to be sustainable it is important that staff involved are regularly informed and reminded of the protocol in place for sending urgent microbiology specimens and that regular audit is implemented to ensure a high quality service is maintained.

\section{References}

None 